\title{
Hacia una cultura neurodidáctica de la evaluación. La percepción del alumnado universitario
}

\author{
María Amparo Calatayud Salom *
}

Resumen. Hoy por hoy, los avances de las neurociencias están aportando un cambio de paradigma en la educación y, por supuesto también, en la evaluación. Están surgiendo evidencias de que una nueva evaluación es necesaria y posible. El presente artículo propone la necesidad de potenciar una cultura evaluativa asentada en los principios neurodidácticos. Esta nueva forma de evaluar conlleva emplear diversas técnicas de evaluación participativas tanto para el alumnado como para el docente. Técnicas que ayuden a los estudiantes a conocer lo que saben y hasta donde pueden llegar potenciando sus capacidades y aprovechando al máximo sus posibilidades. En esta investigación se presentan no sólo los supuestos teóricos y las implicaciones metodológicas para hacer realidad practicas neuroevaluativas sino también se presentan los resultados cualitativos obtenidos por una muestra de 240 estudiantes universitarios acerca de cómo han sido evaluados. Desde la mirada de los estudiantes podemos constatar que la evaluación vivida dista mucho de los supuestos impregnados por la neurociencia. Constatar esta realidad es necesario para darse cuenta de que ésta ha de cambiar, si queremos realizar prácticas evaluativas de excelencia que estimulen la generación de neurotransmisores que garanticen adecuadas sinapsis en el cerebro de los estudiantes. Los docentes tenemos el reto de ser modificadores cerebrales y la practica evaluativa determina, sin duda, la estructura, la química y la actividad eléctrica del cerebro. Por tanto, impregnar el proceso evaluativo de fundamentos neuroeducativos supone una exigencia y una necesidad hoy en la profesión docente.

Palabras clave: neurociencia, evaluación, neurodidáctica, técnicas evaluativas.

\section{POR UMA CULTURA NEURODIDÁTICA DE AVALIAÇÃO. A PERCEPÇÃO DO ALUNO UNIVERSITÁRIO}

Resumo. Hoje, os avanços na neurociência estão contribuindo para uma mudança de paradigma na educação e, naturalmente, também na avaliação. Estão surgindo evidências de que uma nova avaliação é necessária e possível. Este artigo propõe a necessidade de potencializar uma cultura avaliativa baseada em princípios neurodidáticos. Essa nova forma de avaliar envolve o emprego de diversas técnicas de avaliação participativas, tanto para o corpo discente quanto para o docente. Trata-se de técnicas que ajudam os estudantes a conhecer o que sabem e até onde podem ir, aprimorando suas capacidades e aproveitando ao máximo suas possibilidades.

\footnotetext{
* Universidad de Valencia (UV), España.
} 
Esta pesquisa apresenta não apenas os pressupostos teóricos e as implicações metodológicas para a concretização das práticas neuroavaliativas, mas também os resultados qualitativos obtidos por uma amostra de 240 estudantes universitários sobre a forma que foram avaliados. Do ponto de vista dos alunos, podemos observar que a avaliação praticada está distante dos pressupostos impregnados pela neurociência. É necessário constatar essa realidade para perceber que ela precisa ser modificada se quisermos realizar práticas avaliativas de excelência que estimulem a geração de neurotransmissores, garantindo sinapses adequadas no cérebro dos estudantes. Os professores têm o desafio de serem agentes modificadores cerebrais e a prática avaliativa determina, sem dúvida, a estrutura, a química e a atividade elétrica do cérebro. Portanto, impregnar o processo de avaliação de fundamentos neuroeducacionais é atualmente um requisito e uma necessidade na profissão docente.

Palavrsa-chave: neurociência; avaliação; neurodidática; técnicas avaliativas.

\section{TOWARDS A NEURODYDACTIC CULTURE OF EVALUATION. THE PERCEPTION OF THE UNIVERSITY STUDENT}

Abstract. Today, advances in neuroscience are bringing a paradigm shift in education and, of course, in evaluation. Evidence is emerging that a new evaluation is necessary and possible. This article proposes the need to strengthen an evaluative culture based on neurodynamic principles. This new way of evaluating involves employing various participatory evaluation techniques for both the student body and the teacher. Techniques that help students know what they know and how far they can go by enhancing their abilities and making the most of their possibilities.

This research presents not only theoretical assumptions and methodological implications for making neuroevaluative practices a reality, but also presents the qualitative results obtained by a sample of 240 university students about how they have been evaluated. From the students' point of view, we can see that the evaluation is far from the assumptions impregnated by neuroscience. To verify this reality is necessary to realize that it has to change if we want to carry out evaluative practices of excellence that stimulate the generation of neurotransmitters that guarantee adequate synapses in the students' brains. Teachers have the challenge of being brain modifiers and evaluative practice determines, without doubt, the structure, chemistry and electrical activity of the brain. Therefore, impregnate the evaluation process of neuroeducational foundations is a requirement and a need today in the teaching profession.

Keywords: neuroscience, evaluation, neurodidactic, evaluative techniques. 


\section{A MODO DE INTRODUCCIÓN}

En los últimos 20 años, las neurociencias han experimentado un gran desarrollo. Si bien, es una disciplina relativamente reciente, aporta cambios relevantes y significativos que han originado en el plano teórico una verdadera revolución en la cultura evaluativa.

Los avances en las Neurociencias nos han posibilitado el acceder con una mayor exactitud a cómo aprende el cerebro. Hoy por hoy, los progresos de las neurociencias están aportando un cambio de paradigma en la educación y, por supuesto, también en la evaluación. Están surgiendo evidencias de que una nueva evaluación es necesaria y posible. Estas evidencias son de vital importancia para poder mejorar los procesos de enseñanza y aprendizaje y para realizar una evaluación que se adapte a los intereses y particularidades de cada estudiante.

Recientes investigaciones (Kalbfleish, 2012; Wassermann y Zambo, 2013) consideran que el objetivo de la Neurodidáctica radica en responder a la diversidad de estudiantes desde el aula, es decir, desde un sistema inclusivo, creando sinapsis, enriqueciendo el número de conexiones neurales mediante interacciones que determinen el cableado neuronal y promuevan la mayor cantidad de interconexiones del cerebro. Indudablemente, si esta disciplina se ocupa de estudiar la optimización del proceso de enseñanza y aprendizaje, basado en el desarrollo del cerebro y favorece a su vez el que se aprenda con todo el potencial cerebral que poseemos, presenta, por tanto, una incidencia transcendental en el proceso evaluador dado que a cada estudiante se le debería de evaluar según su ritmo de aprendizaje y atendiendo a su idiosincrásica particular. Pero, ¿es esto lo que hacemos los profesores?

Indudablemente, si hiciéramos una reflexión sincera y honesta nuestra respuesta seria negativa. La mayoría de las veces, el sistema de evaluación que utilizamos es cerrado, basados en datos, números, etc. Además, desconocemos si el estudiante es capaz de relacionar, transferir la información en conocimiento, si es capaz de trasladar lo que ha aprendido a situaciones distintas a aquellas que generaron el aprendizaje original, etc.

Es por ello que atender e introducir los supuestos de la Neurociencia nos puede ayudar a dar un giro no sólo en la concepción de la evaluación sino también en su praxis evaluativa. Supone practicar una evaluación que forme al estudiante, se adapte a sus peculiaridades y forme también al docente. 


\section{CULTURA EVALUATIVA SIN APORTACIONES DE LA NEUROCIENCIA}

Obviamente reflexionar acerca de cómo enseñar mejor, cómo ayudar a que el aprendizaje de los discentes sea de mayor calidad, cómo favorecer el cambio y el desarrollo profesional e institucional, etc. Son cuestiones que necesitan de la evaluación entendida como instrumento de aprendizaje y mejora profesional. Pero, cuando se ha trabajado esta cuestión con profesores y profesoras, la mayoría de ellos y de ellas han incidido en la función de acreditación, segregación, calificación, medición de los resultados finales de aprendizaje, etc. en vez de incurrir en la función de la evaluación como medio para ayudar al alumnado a aprender y a ser cada vez más autónomo.

Mi pregunta al respecto es bien sencilla y complicada a la vez ¿por qué?, ¿cuáles son, en el 2018, los condicionantes y las condiciones que inciden en una cultura de la evaluación equiparable a calificación y que no tiene en cuenta las aportaciones de la neurodidáctica?

Indudablemente, existen muchos factores que están determinando el que aún hoy exista una cultura evaluativa como instrumento sancionador, calificador y sin contar con los avances de la neurodidáctica. A continuación comentaré algunos de los aspectos más relevantes:

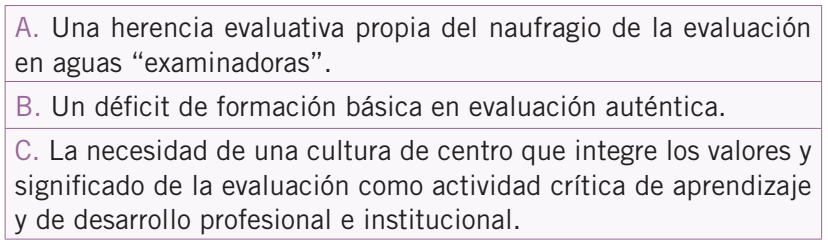

A. Una herencia evaluativa propia del naufragio de la evaluación en aguas "examinadoras"

Durante mucho tiempo e incluso me atrevería a decir que, en muchos de nuestros Institutos de Educación Secundaria de España, aún persiste la evaluación dirigida casi exclusivamente a medir resultados finales de aprendizaje. La función social de la evaluación ha sido mucho más determinante que la función educativa, de análisis de los procesos, de diagnóstico, de comprensión, diálogo, retroalimentación y de participación activa del alumnado en el proceso evaluativo.

Esta concepción de la evaluación vinculada directamente con la valoración de los resultados del aprendizaje de los estudiantes y mediatizada por una racionalidad propia de la perspectiva tecnoburocrática del currículum. Y en la que la evaluación se sitúa en un pensamiento restringido: sumativo y cuantificador, ha dado lugar a una Tradición denominada como Examinadora. 
Tradición evaluativa que se fundamenta en una serie de supuestos epistemológicos, entre los que destacamos: el curricular, el psicológico, el paradigmático, el socio-antropológico, etc. A continuación, se comentaran algunos de estos supuestos que distan mucho de los fundamentos neurodidácticos, puesto que ello facilitará entender el sentido que persigue este planteamiento evaluativo que, por desgracia, aún impera en nuestro contexto.

\section{Cuadro-resumen de los supuestos epistemológicos que vertebran}

la Tradición Examinadora de la evaluación

1. Concepción científica del curriculum: la valoración tecnocrática de la evaluación.

2. Evaluación basada en la comprobación de los objetivos operativos (propuesta evaluadora de Tyler), evaluación por objetivos.

3. Psicología conductista. Evaluación conductual: evaluación rigurosa de los resultados inmediatos y observables en educación.

4. La incidencia de las taxonomías como instrumentos de evaluación.

5. Incursiones de la psicometría. Modelo de tests criteriales y tests estandarizados.

6. Sinónimos de evaluación como: calificación, medición, examen. La evaluación se convierte en el acto de examinar, calificar a los alumnos.

7. Monismo metodológico, enmarcados en criterios de validez y fiabilidad estadísticos.

8. Cuantificación de los datos de evaluación.

9. Creencia en la objetividad y neutralidad de la evaluación.

10. Evaluación orientada a la comprobación, desde un planteamiento reduccionista, reproductivo, memorístico, etc.

11. Tradición caracterizada por la obsesión de equiparar la evaluación de los alumnos con la medición de capacidades intelectuales.

12. Postulados propios de la tradición clásica con claras evidencias al normotipo estadístico; situando a los alumnos en una determinada "grading curve", configurada por el \% de discentes que forman cada uno de los grupos: a) grupos de alumnos con resultados buenos; b) con resultados bajos y c) con resultados medios.

13. Concepción reproductiva de la educación.

14. Ideología de control. Apoyada en una epistemología claramente racionalista, pragmática, eficientista, etc.

15. Tradición que suele destinar la información sobre la evaluación de los alumnos hacia fines claramente certificadores, selectivos, jerarquizadores, etc.

16. Control burocrático del producto final-rendimiento académico del alumno.

Supuestos que como hemos tenido ocasión de investigar, en varios estudios realizados, tienen unas determinadas implicaciones en la práctica evaluativa (Calatayud, 2000; 2000a). A continuación, se describirán algunas de las características más importantes que vertebran esta tradición evaluativa:

- Prevalece la observación de los resultados más que la valoración de los procesos.

- Sólo se evalúan contenidos conceptuales.

- Evaluación comprobativa (si los objetivos previstos se han alcanzado). 
- Evaluación continua con carácter sumativo.

- La evaluación como instrumento coercitivo, punitivo,...

- Evaluación finalista.

- Uso casi exclusivo del examen.

- El culpable del fracaso escolar es el alumno.

- Objeto de evaluación es exclusivamente el discente.

- No se negocian los criterios de evaluación con los alumnos.

- La recuperación es la repetición del examen.

- Evaluar es sinónimo de calificar, medir,...

- Se menosprecia el uso de la autoevaluación como elemento de reflexión, desarrollo y madurez.

- La referencia al control/examen para tomar las decisiones de promoción.

- En las sesiones de evaluación se "cantan" las notas.

- No se asume la importancia del error como fuente de aprendizaje.

- Evaluación para detectar lo negativo.

- Evaluación puntual.

- Evaluación con carácter explícito.

- Se les otorga más peso a las evaluaciones de tipo formal.

- Se ignora el valor de la evaluación del proceso de enseñanza y aprendizaje como instrumento de mejora de la Institución Escolar.

B. Un déficit de formación básica en evaluación auténtica

A pesar de la formación pedagógica que el profesorado de educación secundaria ha recibido a través del Master de Formación de Profesorado de Educación Secundaria, se constata un déficit de formación en los supuestos que aportan las Neurociencias.

El profesorado de secundaria al carecer de formación en relación con el tema que nos ocupa, tiende a perpetuar las prácticas que durante su etapa de estudiante le fueron aplicadas por sus respectivos profesores y profesoras. Se cree que la evaluación es un tema "fácil", dado que elaborar un examen y calificarlo puede hacerlo cualquier persona.

En estos momentos, la preocupación por la calidad en educación y por la evaluación son cuestiones de máxima actualidad que comparten la mayoría de los países desarrollados, sin embargo, la formación pedagógica del docente en materia de evaluación auténtica deja mucho que desear. (Calatayud, 2004; 2004a). 
Estudios realizados al respecto avalan el reclamo que existe por parte del profesorado de la necesidad de recibir una mayor formación y preparación para desarrollar mejor la práctica de la evaluación de sus estudiantes. Como demuestra una investigación ya realizada por el profesor Castillo Arredondo (2004) sobre la práctica evaluadora, los docentes siguen manifestando abiertamente que "carecen de formación pedagógica para evaluar".

A su vez, el profesorado también manifiesta en otras investigaciones que, como consecuencia del fenómeno de la globalización en educación, la sociedad del conocimiento, etc. necesita una determinada formación dado que educar en un mundo plural, en una sociedad democrática y para la ciudadanía reclama nuevas y diversas demandas formativas. Por ejemplo, formación en interculturalidad, en nuevas tecnologías, en estrategias para aprender a aprender, en el tratamiento de la diversidad, nuevas formas de organizar el currículo, nuevos sistemas de funcionamiento y estructura de los centros educativos, etc.

Demandas formativas a las que se les ha de dar respuesta desde las distintas entidades y administraciones educativas competentes. Pero quizás, antes de todo ello, sería necesario incidir en formar al docente en materia de evaluación auténtica ¿Por qué esta afirmación? La respuesta es bien sencilla, porque la evaluación guía la enseñanza y porque además ésta se convierte en el requisito previo a cualquier actividad educativa, dado que el buen uso de la misma ayuda a conocer cómo enseñar mejor a nuestros estudiantes en el aula.

Por tanto, es fundamental formar al docente en evaluación para que deje de considerarla como un hecho puntual en el proceso de enseñanza, con la finalidad última de certificar, a través de una calificación, el nivel de aprendizaje alcanzado por los alumnos, equiparándola a conceptos como medición, clasificación, calificación, etc. Funciones que ya no representan las acciones primordiales a las que ha de servir la evaluación como nos señalan los principios de la neurodidáctica.

Hoy el énfasis se encuentra en el aprendizaje de los estudiantes y en la evaluación como una valiosa herramienta capaz de dar respuesta a las necesidades de las distintas personas implicadas en el proceso educativo y de ofrecer una información detallada sobre el desarrollo del mismo. En este sentido, la evaluación se sitúa al servicio del aprendizaje, dado que éste es el mejor modo de ayudar a los estudiantes a aprender y a ser capaces de valorar el propio progreso académico y el desarrollo de sus capacidades personales. Para ello, la evaluación ha de dejar de representar una acción al margen del proceso educativo para convertirse en una situación habitual en la actividad escolar, en un elemento verdaderamente integrado en el proceso de enseñanza y aprendizaje (Allen, 2000). 
Para comprender la evaluación como instrumento de aprendizaje que está al servicio tanto de quien aprende como también del profesor para ayudarle a que el alumno aprenda mejor, se necesita formación pedagógica que no sólo incida en los planteamientos teóricos de la evaluación, en la normativa, etc. sino en lo que es más importante, en facilitar al profesorado una serie de estrategias e instrumentos que ayuden verdaderamente a llevar a la práctica diaria del aula los supuestos evaluativos inmersos en la cultura neuroevaluativa.

C. La necesidad de una cultura de centro que integre los valores y significado de la evaluación como actividad crítica de aprendizaje y de desarrollo profesional e institucional

A esta premisa se le concede un valor especial dado que si en el centro no existe una predisposición y unos mínimos significados compartidos por las personas que interactúan en la institución escolar, unos valores y creencias sobre la evaluación como instrumento para mejorar los procesos de enseñanza y aprendizaje, difícilmente la evaluación será entendida como ayuda al aprendizaje y elemento útil para mejorar la enseñanza (currículum, intervención docente, selección y uso de materiales curriculares, etc.).

En los últimos años se le ha concedido una importancia extraordinaria 74 a la cultura organizativa del centro escolar como elemento clave para comprender lo que ocurre en las instituciones y para cambiarlas. Para González y Santana (1999) la cultura es el "halo que impregna todas y cada una de las diferentes facetas de la organización" (pág. 325).

Existen infinidad de definiciones de cultura, lo cual refleja una pluralidad de concepciones existentes en el campo educativo, que remiten a su vez a diversas formas de concebir e investigar el fenómeno de la cultura en la organización.

A pesar de esta gran diversidad de definiciones lo que éstas manifiestan, en general, es que es necesario comprender las culturas para entender el funcionamiento de las escuelas, para saber qué ocurre y por qué, para entender los procesos organizativos y el desarrollo escolar. La cultura desarrollada en el centro constituye una dimensión organizativa que impregna a los diversos modos de actuar en la organización. Por tanto, los cambios que se quieran realizar en las prácticas evaluativas necesariamente requerirán de un cambio en la cultura organizativa. Cultura que la entendemos como el conjunto de creencias, supuestos, normas, formas de actuar, etc. que poco a poco se van construyendo en la realidad del centro, siendo asumida y compartida por los miembros que conforman la organización. Es cómo si hiciéramos mención a las señas de identidad que son las que radiografían y hacen distinta a una institución escolar de otra. Concretamente para algunos autores como 
González (1994), Rossman y Otros (1988), la cultura de la organización es el modo con el que hacemos las cosas las personas que conviven en una misma institución. En cambio, para otros autores, la cultura viene a ser las ideas y teorías que se utilizan colectivamente para interpretar y dar sentido a la realidad organizativa (Sackman, 1991; Smircich, 1983). Por lo tanto, la cultura provee a las personas que integran la institución de un marco de referencia para interpretar los sucesos, así como también para actuar de modo apropiado y correcto según sea la situación. Desde estas premisas hablar de cultura supone mencionar creencias, supuestos, convicciones que los docentes y la comunidad educativa poseen en relación con la enseñanza, el aprendizaje de los estudiantes, el funcionamiento organizativo del centro, la evaluación, etc. Además, la cultura también incluye las relaciones que se establecen entre los distintos miembros de la comunidad educativa, el sistema de comunicación, el tipo de colaboraciones que se producen entre los profesores, etc. (Sarasola, 2004, Marchesi y Martín, 2000).

En parte todas las escuelas son iguales pero, por supuesto, también son diferentes, entre otras razones, porque la interpretación de los significados depende de los miembros que interactúan en la organización escolar. Así de claramente nos lo explicita Pérez Gómez (2010) cuando afirma que "cada escuela configura su propia forma específica de establecer los intercambios personales y curriculares, y aunque pueden encontrarse elementos comunes que se repiten en las circunstancias más diversas, siempre actuarán de manera singular" (pág. 154). Entre otras razones porque la cultura de cada centro se configura en un contexto, tiempo y espacio concreto y lo que es más significativo con unas personas determinadas. Por lo tanto, representa de algún modo un conjunto de significados que da sentido y subyace al modo de funcionar de una organización (Coronel, 2002)

Ahondando algo más en estas ideas el profesor Coronel (2002), matiza que cada organización presenta una cultura determinada porque cada centro y, en concreto, los miembros que integran la institución responden de distinta manera a cuestiones que tienen que ver, por ejemplo, con: los objetivos de la institución y su uso en la toma de decisiones, en relación con la socialización (cómo se socializan los nuevos miembros de la institución), en relación con el liderazgo (qué espera la organización de los líderes), en relación a la información (cómo se genera y es diseminada), etc. En relación con la evaluación, añadiría que cada centro responde de manera diferente a cuestiones que tienen que ver, por ejemplo, con: ¿qué significa evaluar?, ¿para qué evaluamos a nuestros estudiantes, etc.? 


\section{LA NEURODIDACTICA: HACIA UNA NUEVA FORMA DE HACER EVALUACIÓN}

Los avances de la neurociencia inciden en una concepción de la evaluación que apuesta por insertarla en el proceso de aprendizaje, primar la función de diagnóstico, de regulación y adaptación de la planificación del proceso de enseñanza y aprendizaje a las necesidades y dificultades de los estudiantes. Además, incide en que es fundamental emplear diversas técnicas de evaluación, igualmente activas y participativas, no sólo para que el docente las conozca, sino también para que los estudiantes, protagonistas de sus propios aprendizajes puedan tener conocimiento de aquello que saben y hasta dónde pueden llegar, generando en ellos una mentalidad de crecimiento a través de la evaluación.

Concepción evaluativa basada en las directrices de la perspectiva práctica-critica y rotundamente vinculada a los procesos, así como en plantear el carácter ideológico de la evaluación, bajo determinantes constructivistas y de pensamiento crítico. Evaluación que debe ser antes que nada un medio para formar al estudiante en su progreso y desarrollo del proceso de enseñanza y aprendizaje. Situándonos en un enfoque más complejo de la evaluación: formativo-cualitativo. Pensamiento propio de la Tradición Auténtica de la evaluación que se fundamenta en los supuestos de la Neurodidáctica.

Dicha tradición se asienta en una serie de supuestos epistemológicos, entre los que destacamos: el curricular, el psicológico, el paradigmático, el socio-antropológico, etc. A continuación, se comentan algunos supuestos propios de este planteamiento evaluativo.

Cuadro-resumen de los supuestos epistemológicos que vertebran la Tradición Auténtica de la evaluación

1. Filosofía hermenéutica-crítica.

2. Incorporación de enfoques ambientalistas en psicología y análisis ecológicos de educación.

3. Las implicaciones del modelo mediacional en su doble vertiente alumno y docente.

4. Raíces progresistas-humanistas. La recuperación del valor educativo de la evaluación.

5. El legado de los modelos alternativos de evaluación.

6. Psicología cognitiva-constructivista. Evaluación con rasgos claramente diagnósticos, significativos, transferibles, interactivos,...

7. Evaluación unida a términos como diálogo, investigación, crítica, análisis, reflexión, etcétera.

8. Evaluación de los efectos secundarios, de lo singular, lo imprevisible.

9. Evaluación de capacidades frente a evaluación de conductas.

10. Variedad metodológica y uso de métodos cualitativos.

11. Relativización de la objetividad y neutralidad científica de la evaluación.

12. Carácter axiológico de la evaluación. 
13. Evaluación asentada en posicionamientos de autoevaluación, informales, idiográficos, de énfasis en lo procesual, holísticos, etc.

14. Ideología sobre evaluación encauzada hacia posicionamientos más pedagógicos: evaluación como instrumento de aprendizaje y la evaluación como instrumento de mejora de la enseñanza.

15. La influencia de la teoría de resistencia de la educación. El adentrarse en la estructura profunda de la evaluación.

16. Tradición que suele destinar la información de la evaluación de los alumnos hacia fines de diagnóstico, de orientación, de incremento de la motivación, de mejora, etc.

17. Tratamiento democrático de la información en la interpretación de los procesos de evaluación: integración y participación social como finalidad educativa general.

Supuestos que como se ha tenido ocasión de investigar en varios estudios realizados, tienen unas determinadas implicaciones en la práctica evaluativa de los docentes (Calatayud, 1998; 2000; 2000a). A continuación, se describirán algunas de las características más importantes.

- Una evaluación en la que existe un mayor predominio de la evaluación informal frente a la evaluación formal.

- Evaluación significativa, diversificada, diagnóstica, procesual, contextualizada, coherente, cualitativa, ideografíca y transferible.

- Evaluación integrada en el proceso de enseñanza y aprendizaje.

- Evaluación continua desde un enfoque constructivista.

- Mayor potenciación de informes cualitativos en la evaluación del aprendizaje del alumnado.

- Evaluación que plantea la necesidad de evaluar también lo positivo y no sólo lo negativo.

- Evaluación que incorpora la autoevaluación y la coevaluación.

- El trabajo del alumno como principal fuente en los procesos de evaluación del aprendizaje.

- Se evalúan tanto contenidos conceptuales, procedimentales y actitudinales.

- Evaluación con carácter implícito.

Por tanto, se trata de una evaluación que está integrada dentro del proceso de enseñanza y aprendizaje y no separada o desconectada de él. Planteamiento que se opone al que concibe la evaluación como una práctica que se efectúa al final de un periodo más o menos prolongado de enseñanza, o al término de la realización de alguna unidad temática mediante un acto formal explícito de comprobación, como es el poner una prueba o realizar un examen. Se intenta que la evaluación no sólo sea un acto que conduzca a poner una nota sino que sobre todo, ésta se entienda como un proceso 
de comprensión, de diálogo, de motivación hacia el aprendizaje tanto del profesor como de los alumnos. Una evaluación, a fin de cuentas, como dice Fernández Pérez (1986) que eduque, que nos enseñe "qué es debido a qué", una evaluación formativa y no únicamente sumativa. En este sentido, una evaluación para que sea realmente educativa, emancipadora e iluminativa tiene que ser concebida como:

- Una reflexión sobre los procesos de enseñanza y aprendizaje, las finalidades que los orientan y los condicionantes que confinan y restringen su desarrollo.

- Un proceso continuado de investigación que no puede entenderse únicamente como referida al aprendizaje alcanzado por los estudiantes, sino que tiene que ser una valoración de todo el proceso y de todos los elementos que en él participan (docente, alumnos, asignatura, estrategias metodológicas, etc.). Una evaluación tanto de los conocimientos, actitudes y habilidades adquiridos por los discentes y en los que la evaluación no se reduce al momento final del proceso, sino que se realiza en distintos momentos y con diversas estrategias.

Desarrollar la evaluación desde los principios neurodidácticos requiere de unos requisitos, algunos de ellos son:

- Una evaluación que esté integrada en el proceso de enseñanza y aprendizaje.

- Una evaluación que incida en el proceso, formativa, procesual, continua, etc.

- Una evaluación que se haga con la finalidad básica de obtener información del alumnado, del proceso y del contexto de aprendizaje, con el fin de mejorarlos. Que sea en definitiva, útil.

- Una evaluación holística. Evaluación de conceptos, procedimientos, actitudes competencias.

- Una evaluación que reconozca los esfuerzos realizados por los alumnos.

- Una evaluación que sea respetuosa con la persona.

- Una evaluación que utilice diversidad de instrumentos para evaluar el aprendizaje del alumnado.

- Una evaluación dentro de lo posible que sea ética, basada en compromisos explícitos que aseguren la cooperación y la aceptación de las personas implicadas. En este sentido, los criterios de evaluación serán públicos, explicitados y negociados con los estudiantes. 
- Una evaluación que sea coherente con los contenidos, la enseñanza y las actividades realizadas en clase ("Dime cómo enseñas y te diré cómo evalúas).

- Una evaluación que presente distintos niveles de dificultad.

- Una evaluación centrada en si el estudiante es capaz de relacionar lo aprendido con otros conocimientos, si es capaz de trasladarlo a situaciones distintas a aquellas que generaron el aprendizaje original.

- Una evaluación que favorezca la reflexión, la innovación, la creatividad así como el pensamiento crítico y lateral.

- Una evaluación que fomente el trabajo cooperativo y las relaciones socio-afectivas.

\section{DISEÑO DE LA INVESTIGACIÓN}

El objetivo general de esta investigación es analizar las percepciones del alumnado universitario en el curso académico 2017-18 acerca de cómo han sido evaluados en sus años de escolaridad. Se pretendía constatar si la evaluación vivida distaba o no mucho de los supuestos impregnados por la neurociencia. Aunque la investigación es más amplia, en este artículo sólo se va incidir en tres cuestiones de las 10 que se plantearon en este estudio.

Las tres cuestiones a las que aludimos son las siguientes:

- Significado de la evaluación vivida.

- Procesos mentales implicados en la evaluación vivida.

- Instrumentos con los que se les ha evaluado.

\section{Población}

Han participado en este estudio 240 estudiantes de primer curso del Grado de Magisterio de la Universidad de Valencia (España).

\section{Instrumento}

Se diseñó un cuestionario abierto para indagar cuestiones relativas a la percepción acerca de la evaluación vivida. Desde la mirada de los estudiantes universitarios podemos constatar si la evaluación vivida dista o no mucho de los supuestos impregnados por la neurociencia. 
El cuestionario fue previamente validado por un grupo de expertos (inspección educativa, asesores de formación del profesorado y profesorado universitario de la Universidad de Valencia). Se confirmó que las preguntastema eran adecuadas para explorar algunas ideas relativas al objeto de estudio.

A continuación se describen las valoraciones más destacadas por los participantes que han intervenido en este estudio.

\section{DISCUSIÓN: LA PERCEPCIÓN DEL ALUMNADO UNIVERSITARIO}

Después de analizar las respuestas de la muestra participante en esta investigación, se agruparon de forma sintética los comentarios más relevantes de los estudiantes en relación a las tres preguntas planteadas. Para el tratamiento de los datos se hizo un análisis cualitativo, organizando la información obtenida en las distintas categorías establecidas según su grado de frecuencia.

A continuación, se describen algunas de las categorías más importantes en relación con las tres cuestiones planteadas:

- Significado de la evaluación vivida.

- Procesos mentales implicados en la evaluación vivida.

- Instrumentos con los que se les ha evaluado.

A. Significado de la evaluación vivida:

Las respuestas a esta pregunta inciden mayoritariamente en una concepción de la evaluación como:

- Herramienta que se usa para comprobar el nivel de asimilación de los conceptos.

- Examen que se realiza de forma objetiva sobre aspectos concretos de una asignatura.

- Es el procedimiento mediante el cual el docente examina al estudiante para comprobar que conocimientos ha adquirido y calificarlos.

- Un conjunto de pruebas que se le pasan al alumnado para conocer si ha adquirido los conocimientos.

- Una nota que plasma un resultado final. 
- Método mediante el cual se averigua si un estudiante ha aprendido lo que se esperaba.

Después de estas concepciones le siguen en mucha menor medida, la idea de la evaluación como:

- Observar y conocer los conocimientos que tiene un estudiante.

- La valoración de conocimientos, actitud y rendimiento de una persona.

- La forma de ver la evolución de un estudiante y ver si está aprendiendo.

- Proceso que permite observar en qué punto del proceso de aprendizaje está el alumno para saber si sigue el ritmo adecuado de la clase.

\section{B. Procesos mentales implicados en la evaluación vivida}

En este caso, las respuestas se organizaron de forma exclusiva en las siguientes afirmaciones: memorización, repetición, concentración, organización, empollar, vomitar todo lo estudiado. Algunos estudiantes hablan incluso de vómito cognitivo. En un índice más bajo de incidencia se presentan los siguientes procesos mentales implicados en la evaluación: pensar, relacionar conceptos, transferir los conocimientos, reflexión, comprender, entender, razonar, elaborar mapas conceptuales y aprender.

\section{Instrumentos con los que se les ha evaluado}

Según los encuestados, mayoritariamente han sido evaluados a través de exámenes (tipo test, de desarrollo y orales) controles, trabajos y por su apellido. Y en menor medida, a través de tareas de clase, participación, revisión del cuaderno, observación, debate en el aula, entrevistas, deberes, trabajo diario, libreta, comportamiento y actitud, asistencia, reuniones consensuadas para evidenciar el aprendizaje realizado y portafolio.

\section{CONSIDERACIONES FINALES. HACIA INSTRUMENTOS NEUROEVALUATIVOS}

Después de los resultados extraídos en esta investigación, una de las cuestiones que ha quedado suficientemente clara, es que asistimos a una cultura evaluativa vivida dentro de las coordenadas de lo que hemos denominado Tradición Examinadora de la evaluación. 
Un análisis de la evaluación vivida por los estudiantes encuestados conlleva la necesidad de reinventar métodos de evaluación acordes con los postulados de la Neurociencia. Debemos de modernizar muchos aspectos en educación para generar y transferir conocimiento, para aprender de otra forma, para enseñar y evaluar de forma diferente a nuestros estudiantes y eso exige transformar, perfeccionar e innovar a través de las aportaciones de la neurodidáctica.

En estos momentos, deberíamos por empezar a reinventar instrumentos neuroevaluativos que posibilitaran el acceso a una evaluación que facilitara la mejora de los aprendizajes de los estudiantes, potenciando al máximo sus capacidades y sus posibilidades, utilizando el error como fuente de aprendizaje. Algunos de estos instrumentos pueden ser los siguientes:

- Actividades prácticas que pretendan fomentar en los estudiantes la capacidad de juicio autónomo, de disentiry discrepar, de buscar soluciones personales a los problemas. No se puede caer en la trampa, de la respuesta convergente y correcta. Desde esta visión, se han de proponer a los estudiantes un sistema de trabajo que les permita desplegar su capacidad de comprensión y reflexión sobre las ideas expuestas en clase y las lecturas realizadas.

- Pruebas de aprendizaje con el objeto de conocer si los estudiantes comprenden y transfieren los contenidos fundamentales de cada uno de los temas. Instrumentos que recogen, por un lado, la sistematización de los conocimientos y, por otro, su organización interna. Para ello es necesario el que trabajen a partir de mapas conceptuales, rúbricas, etc.

- Trabajos de investigación Se pretende que cumplan la misión de indagación, de investigación, etc. Se valora el rigor en las argumentaciones, la coherencia, la capacidad de reflexión crítica, la utilización de bibliografía adecuada, el uso del lenguaje correcto, la presentación, etc.

- La autoevaluación del estudiante como estrategia formativa, de responsabilización y reflexión del proceso de aprendizaje. Si en teoría el protagonista de la educación es el alumno, por lógica, no se le puede negar en la práctica que participe en su proceso de enseñanza y aprendizaje de una manera activa, así como también, en el proceso de evaluación a través, por ejemplo, de la elaboración de instrumentos evaluativos. Concretamente para Boud (1995) se habla de autoevaluación cuando los estudiantes toman la responsabilidad de controlar y hacer juicios sobre aspectos de su propio aprendizaje. Este proceso sugiere, animar 
a los estudiantes a buscar por sí mismos y en otras fuentes la determinación de criterios que deberán de utilizar para juzgar su propio trabajo, en lugar de dejarse llevar y ser dependientes de los criterios elaborados por sus profesores. En este sentido, la autoevaluación es una forma de implicar al estudiante en su propio proceso formativo, ya que fomenta la reflexión y la autocrítica sobre el mismo proceso.

- El portafolio, instrumento para la evaluación formativa del estudiante. El portafolio es un método de evaluación del alumnado, alternativo a los métodos tradicionales. Viene a ser como una colección de trabajos, actividades, etc. que el estudiante ha realizado durante un curso. Quizás, lo más importante de esta selección de actividades radique en las siguientes premisas: deben de tratar de evidenciar los esfuerzos realizados por el estudiante, la valoración del trabajo conseguido (¿qué sabía?, ¿qué sé yo ahora?, ¿cómo lo he aprendido?, en relación a contenidos tanto del ámbito conceptual, procedimental y actitudinal, así como las competencias aprendidas), cuáles han sido las mejores ideas, los logros conseguidos en los distintos ámbitos de conocimiento y, sobre todo, el portafolio debe de estar compuesto por aquellas actividades que han permitido al estudiante tanto la posibilidad de valorarse más a sí mismos, como de sentirse más seguros de sí mismos.

Indudablemente, esta forma de practicar la evaluación conlleva necesariamente una perspectiva también distinta de pensar y desarrollar el proceso educativo. Implica una metodología basada en el aprendizaje constructivo, en el fomento de la creatividad, la reflexión, la colaboración, la actividad, la participación, etc. que posibilite al alumno progresar en su madurez y conseguir un equilibrio personal y una integración social.

- Las rúbricas. Este instrumento nos va a facilitar el aprendizaje, dado que determina qué se espera y a su vez, constituye una guía de evaluación tanto para el alumnado como para el profesorado.

- Diario de aprendizaje. Instrumento que desarrolla la metacognición en el alumnado. Cada estudiante reflexiona sobre lo aprendido, qué dificultades ha tenido, qué puede mejorar, etc.

- Dianas de evaluación. Que nos ayudan a averiguar cómo está aprendiendo el estudiante, especificando de forma clara qué se quiere evaluar y los indicadores que se van a utilizar. 
Como conclusión señalar, que si bien educar es modificar el cerebro, y para tratar de influenciar en el cerebro la educación ha de no sólo conocer, sino aplicar los avances de las neurociencias. Avances que están originando cambios importantes en el arte de enseñar y, por supuesto, en el arte de evaluar. Están surgiendo evidencias de que una nueva evaluación es necesaria y posible. A lo largo de este artículo se ha propuesto la necesidad de potenciar una cultura evaluativa asentada en los principios neurodidácticos. Esta nueva forma de evaluar conlleva emplear diversas técnicas de evaluación participativas tanto para el alumnado como para el docente. Técnicas que ayuden a los estudiantes a conocer lo que saben y hasta donde pueden llegar potenciando sus capacidades y aprovechando al máximo sus posibilidades.

A lo largo de este artículo se ha constatado que los docentes tenemos el reto de ser modificadores cerebrales y la practica evaluativa, sin duda, determina la estructura, la química y la actividad eléctrica del cerebro (dime cómo me vas a evaluar y te diré cómo tengo que estudiar). Por tanto, impregnar el proceso evaluativo de fundamentos neuroeducativos, supone una exigencia y una necesidad hoy en la profesión docente.

\section{BIBLIOGRAFÍA}

Allen, D. (2000). La evaluación del aprendizaje de los estudiantes. Barcelona: Paidós.

Boud, D. (1995). Enhancing learning through self assessment.London: Kogan.

Calatayud Salom, M.A (1998). Las tradiciones culturales sobre la evaluación en la Etapa de Educación Primaria. Valencia: Universidad de Valencia.

Calatayud Salom, M.A (2000). "La Evaluación en la Educación Secundaria Obligatoria. Dificultades percibidas y estrategias de superación". Revista de Ciencias de la Educación. Núm 183.

Calatayud Salom, M.A (2000a)."Reflexión de los alumnos de Educación Primaria sobre preconcepciones evaluativas". AA.VV Evaluación como ayuda al aprendizaje. Barcelona: Graó-Laboratorio Educativo.

Calatayud Salom, M.A (2004). "Formación en evaluación educativa. Sí, por favor". Periódico Digital de Información Educativa: Comunidad Escolar. № 755 http: //comunidadescolar.pntic.mec.es/755/ tribuna.html, 2004.

Calatayud Salom, M.A (2004a) "El olvido de la Formación Permanente del Profesorado". Periódico Digital de Información Educativa: Comunidad Escolar. № 742 http: //comunidadescolar.pntic.mec.es/742/ tribuna.html. 2004a

Castillo Arredondo, S. (2004). La práctica evaluadora del profesorado. Primaria y Secundaria. Madrid: Grupo Editorial Universitario

Coronel J.M. (2002). Las culturas organizativas". En M.J. Carrasco y Otros. Organización Escolar. Aspectos Básicos para docentes. Madrid: Grupo Editorial Universitario, 
Fernández Pérez, M. (1986). Evaluación y cambio educativo. El fracaso escolar. Madrid: Morata.

González, M.T.(1994). ¿La cultura del centro escolar o el centro escolar como cultura? En: J.M. Escudero y $\mathrm{M}^{\mathrm{a}} \mathrm{I}$. González (Eds.) Escuelas y Profesores ¿Hacia una reconversión de los centros y la función docente? Madrid: Ediciones Pedagógicas.

González Ma T. y Santana, P. (1999). La cultura de los centros, el desarrollo del currículo y las reformas. En: J.M. Escudero, (Ed), Diseño, desarrollo e innovación del currículo. Madrid: Síntesis.

Kalbfleisch, M.L. (2012). Neurotechnology in education. New York: Taylor and Francis Eds

Marchesi, A. y Martin, E. (2000). Calidad de la enseñanza en tiempos de cambio. Madrid: Alianza Editorial.

Peéez Gomez, A. (2010) "Aprender a educar. Nuevos desafíos para la formación de docentes." Revista Interuniversitaria de Formación del Profesorado, 68. Pp 37-60.

Rossman, G.B.; Corbett, H.D. y Firestone, W. A. (1988). Change and Effectieveness in Schools. New York: State Univ. of New York Press.

Sackman, S.A. (1991). Cultural knowledge in Organizations. Exploring the Collective London: Mind Sage.

Sarasola,M.R.(2004). "Una aproximación al estudio de la cultura organizacional en centros educativos". Archivos Analíticos de Políticas Educativas, 12 (57)

Smircich, L. (1983). “Concepts of Culture and organizational Analysis. Administrative"Science Quarterly. Vol. 28 (1), 1983. Págs. 339-358.

Wassermann,L y Zambo, D. (2013). Early childhood and Neuroscience. New York: Springer. 